\title{
Realization of White LEDs Using Free-Standing Quantum Dot Films
}

\author{
Soyeon Yoon, Ji Hye Oh, Keyong Nam Lee, Young Rag Do \\ Department of Applied Chemistry, Kookmin University \\ 77 Jeongneung-Ro, Seongbuk-Gu, Seoul, 02707, Korea \\ yoonso19@kookmin.ac.kr; ohjh@kookmin.ac.kr; ykn21@kookmin.ac.kr; yrdo@kookmin.ac.kr
}

\section{Extended Abstract}

This study introduces free-standing quantum dot (QD) films using AgIn5S8/ZnS QDs as color-converting materials (CCMs) for remote-type white-light-emitting diodes (WLEDs). QDs are not suitable for the in-cup type of WLEDs as CCMs because they are typically unstable when heat is applied directly to the LED chip. Therefore, we introduce remote-type WLEDs created simply by placing free-standing QD films onto InGaN blue LEDs to reduce the thermal loss from the LED chip.

First, we synthesized Cd-free and broadband-emitting trioctylphosphine (TOP)-capped AgIn5S8/ZnS QDs using a colloidal hot injection method. The AgIn5S8/ZnS QDs show a dominant wavelength of $530 \mathrm{~nm}$, a broad bandwidth of 95 $\mathrm{nm}$ and a moderate photoluminescent quantum yield (PLQY) of 64\% compared to those of rhodamine 6G at $450 \mathrm{~nm}$ [1].

To realize WLEDs, we fabricated free-standing QD films using an electro-spray (e-spray) method, which shows good dispersion in a polymer matrix with a high concentration of QDs compared to the spin-coating method. Uniform and largearea QD films were also fabricated using the e-spray method. The as=prepared AgIn5S8/ZnS QDs were mixed with the poly(methyl methacrylate) (PMMA) polymer matrix in a mixed organic solvent (dimethylformamide/toluene). At the same time, water-soluble polymer, poly(2,3-dihydrothieno-1,4-dioxin)-poly(styrenesulfonate) (PEDOT/PSS) was coated onto indium tin oxide (ITO) glass to fabricate free-standing films by dissolving a PEDOT/PSS interlayer on the water [2].

The mixed QD solution was loaded into an e-spray syringe and e-sprayed onto the PEDOT/PSS-coated ITO glass as a function of the deposition time. For the e-spraying of the mixed QD solution, an electric field of $15 \mathrm{KV} / \mathrm{cm} 2 \mathrm{was}$ applied between the PEDOT/PSS-coated ITO-glass substrate and the e-spray nozzle. Subsequently, the QD-deposited substrate was put into a water bath to dissolve the interlayer of PEDOT/PSS and then picked up as floating, free-standing QD films. The deposition rate of the free-standing QD film was $5 \mu \mathrm{m} /$ hour. The e-spray durations were 3, 4.5, and 6 hours and the films were solvent-annealed to create clear film samples by re-arrangement of the QDs in the matrix [3].

To realize the WLEDs, the free-standing QD films were simply placed onto InGaN blue LEDs, which are an excitation source, and a blue light source, thus creating the WLEDs. The optical properties of the WLEDs show the correlated color temperatures (CCT, K) of 5,500 K, 4,100 K and 3,800 K and luminous efficacy (LE) rates of $76 \mathrm{~lm} / \mathrm{W}, 69 \mathrm{~lm} / \mathrm{W}$, and 65 $\mathrm{lm} / \mathrm{W}$ after 3, 4.5 and 6 hours of the e-spraying of the free-standing QD films, respectively, at an applied current of $60 \mathrm{~mA}$. The color rendering index (CRI, Ra) values of the WLEDs were 71, 61 and 59 after 3, 4.5 and 6 hours of the e-spraying of the free-standing QD films, respectively. The low CRI is due to the lack of reddish color, though this can be improved by using red QDs mixed with green QDs. The results here demonstrate the possibilities of QD films for WLEDs.

\section{References}

[1] S. P. Hong, H. K. Park, J. H. Oh, H. Yang, Y. R. Do, "Comparisons of The Structural and Optical Properties Of OAgins, 2 T-Agins, and C-Agin ${ }_{5} \mathrm{~s}_{8}$ Nanocrystals and Their Solid-Solution Nanocrystals With Zns," J. Mater. Chem., vol. 22, pp. 18939-18949. 2012.

[2] S. P. Hong, H.-Y. Park, J. H. Oh, H. Yang, S.-Y. Jang, Y. R. Do, "Fabrication of Wafer-Scale Free-Standing Quatum Dot/Polymer Nanohybrid Films for White-Light-Emitting Diodes Using An Electrospray Method," J. Mater. Chem. C, vol. 2, pp. 10439-10445. 2014.

[3] H.-Y. Park, J. H. Oh, S. P. Hong, Y. R. Do, "Optical Properties Enhancement of Electrosprayed Quantum Dot/Polymer Nanohybrid Films by a Solvent Vapor Treatment,” Sci. Adv. Mater., vol. 8, pp. 224-230. 2016. 